ARTICLE OPEN

\title{
AXL-associated tumor inflammation as a poor prognostic signature in chemotherapy-treated triple-negative breast cancer patients
}

Giulia Bottai ${ }^{1}$, Carlotta Raschioni ${ }^{1}$, Borbála Székely ${ }^{2}$, Luca Di Tommaso ${ }^{3}$, Attila M Szász ${ }^{2}$, Agnese Losurdo ${ }^{4}$, Balázs Győrffy ${ }^{5,6}$, Balázs Ács ${ }^{2}$, Rosalba Torrisi ${ }^{4}$, Niki Karachaliou ${ }^{7}$, Tímea Tókés ${ }^{2}$, Michele Caruso ${ }^{8}$, Janina Kulka ${ }^{2}$, Massimo Roncalli ${ }^{3,9}$, Armando Santoro ${ }^{4,9}$, Alberto Mantovani ${ }^{9,10}$, Rafael Rosell ${ }^{7,11,13}$, Jorge S Reis-Filho ${ }^{12,13}$ and Libero Santarpia ${ }^{1}$

A subgroup of triple-negative breast cancer (TNBC) shows epithelial-to-mesenchymal transition (EMT) features, which are sustained by the interaction between cancer cells and tumor-associated macrophages (TAMs). In this study, the clinical relevance of 30 EMTrelated kinases and the potential cross-talk with TAMs were investigated in a cohort of 203 TNBC patients treated with adjuvant chemotherapy. The prognostic value of the evaluated markers was validated in two independent cohorts of TNBC patients treated with adjuvant chemotherapy $(N=95 ; N=137)$. In vitro, we investigated the potential synergism between cancer cells and TAMs. We found that the EMT-related kinase AXL showed the highest correlation with the frequency of CD163-positive macrophages $\left(r_{\mathrm{S}}=0.503 ; P<0.0001\right)$. Relapsing TNBC patients presented high expression of AXL $(P<0.0001)$ and CD163 $(P<0.018)$, but only AXL retained independent prognostic significance in multivariate analysis (relapse-free survival, $P=0.002 ;$ overall survival $P=0.001$ ). In vitro analysis demonstrated that $A X L$-expressing TNBC cells were able to polarize human macrophages towards an M2-like phenotype, and modulate a specific pattern of pro-tumor cytokines and chemokines. Selective AXL inhibition impaired the activity of M2-like macrophages, reducing cancer cell invasiveness, and restoring the sensitivity of breast cancer cells to chemotherapeutic drugs. These data suggest that the EMT-related kinase AXL overexpressed in cancer cells has prognostic significance, and contributes to the functional skewing of macrophage functions in TNBC. AXL inhibition may represent a novel strategy to target cancer cells, as well as tumor-promoting TAMs in TNBC.

npj Breast Cancer (2016) 2, 16033; doi:10.1038/npjbcancer.2016.33; published online 2 November 2016

\section{INTRODUCTION}

Triple-negative breast cancer (TNBC) is clinically defined by the lack of expression of estrogen and progesterone receptors, and no overexpression/amplification of HER2. This subgroup of tumors is usually characterized by an aggressive phenotype associated with an increased risk of early recurrence with distant metastasis to visceral organs and poor prognosis. ${ }^{1,2}$ Burgeoning evidence demonstrates that TNBC is a very heterogeneous disease, encompassing different molecular entities. ${ }^{3,4}$ Current treatment approaches are limited to cytotoxic chemotherapy due to the lack of specific therapeutic targets. ${ }^{4,5}$ Even though TNBC patients are initially responsive to chemotherapy, the long-term results are not satisfactory. ${ }^{1,2,4}$ Therefore, the identification of reliable prognostic markers in chemotherapy-treated patients, as well as novel targetable signaling pathways, may allow a better stratification of TNBC patients into different risk groups and the development of novel treatment strategies.
A subgroup of TNBC shows epithelial-to-mesenchymal transition (EMT) features, leading to tumor progression and resistance to chemotherapy. ${ }^{1-5}$ Early tumor recurrences, driven by chemotherapy-resistant cancer cells, represent a prominent cause of poor outcome in TNBC. In this context, EMT allows tumor cells to avoid apoptosis and cellular senescence, increasing tumor cell invasive properties and drug resistance. ${ }^{1,6-8}$

Growing evidence indicates that EMT is induced by different biological mechanisms, and that the mutual interaction between cancer cells and the surrounding microenvironment is a crucial step supporting the entire EMT process. ${ }^{9,10}$ Tumor cells that undergo EMT regulate the phenotype and the activity of nonmalignant stromal cells, particularly tumor-associated macrophages (TAMs), contributing to cancer progression and metastatic spread. ${ }^{11,12}$ TAMs are able to affect the behavior of malignant cells, and to modulate the functions of tumor-infiltrating lymphocytes, overall promoting tumor progression and influencing therapy response. ${ }^{12-19}$ In particular, stromal TAMs with

\footnotetext{
${ }^{1}$ Oncology Experimental Therapeutics, IRCCS Humanitas Clinical and Research Center, Milan, Italy; ${ }^{2}$ 2nd Department of Pathology, Semmelweis University, Budapest, Hungary; ${ }^{3}$ Department of Pathology, IRCCS Humanitas Clinical and Research Center, Milan, Italy; ${ }^{4}$ Department of Oncology and Hematology, IRCCS Humanitas Cancer Center, Milan, Italy; ${ }^{5}$ MTA TTK Momentum Cancer Biomarker Research Group, Hungarian Academy of Sciences, Budapest, Hungary; ${ }^{6}$ 2nd Department of Pediatrics, Semmelweis University, Budapest, Hungary; ${ }^{7}$ Cancer Biology and Precision Medicine Program, Catalan Institute of Oncology, Hospital Germans Trias i Pujol, Barcelona, Spain; ${ }^{8}$ Department of Medical Oncology, Humanitas Oncology Center of Catania, Catania, Italy; ${ }^{9}$ Humanitas University, Milan, Italy; ${ }^{10}$ Department of Immunology and Inflammation, IRCCS Humanitas Clinical and Research

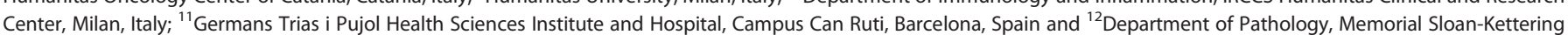
Cancer Center, New York, NY, USA.
}

Correspondence: L Santarpia (libero.santarpia@humanitasresearch.it)

${ }^{13}$ These authors contributed equally to this work.

Received 5 April 2016; revised 10 September 2016; accepted 28 September 2016 
pro-tumor functions, resembling the phenotype of M2-polarized macrophages, have been shown to be capable of inducing EMT, ultimately establishing a positive local feedback loop that sustains cancer cells invasiveness, indirectly influencing the response to chemotherapy. ${ }^{10-19}$ Conversely, conventional chemotherapy has been demonstrated to contribute to the modulation of the tumor microenvironment by inducing the recruitment of TAMs at the tumor site and by reducing therapy efficacy in breast cancer patients. $^{15-20}$

Even though the interaction between cancer cells with mesenchymal traits and TAMs is emerging as a crucial factor in tumor progression and response to therapy, the clinical relevance of this cross-talk in TNBC is still poorly understood. In this study, we first evaluated the expression of several kinases involved in EMT in a large cohort of TNBC patients treated with anthracycline-taxanebased adjuvant chemotherapy. Subsequently, we assessed the clinical relevance of the most significant EMT-related kinase, the receptor $\mathrm{AXL}$, validating its prognostic and functional role in additional independent cohorts of TNBC and by in vitro assays. Finally, we evaluated the correlation between $\mathrm{AXL}$ and TAMs, investigating specific mechanisms by which TNBC cells and macrophages cooperate to influence tumor progression and response to therapy.

\section{RESULTS}

The receptor tyrosine kinase $A X L$ is positively associated with macrophage infiltration in TNBC

To evaluate the potential relationship between cancer cells with mesenchymal features and the presence of TAMs in breast tumor stroma, we selected 30 relevant EMT-related kinases and correlated their expression with the expression of the panmacrophage marker CD68 in a cohort of 203 TNBC patients (Supplementary Tables 1 and 2). We found that AXL was the most significant kinase correlated with the frequency of CD68-positive TAMs in the internal cohort of 203 TNBC patients (Spearman's coefficient, $r_{\mathrm{S}}=0.405$; Bonferroni-adjusted $P=0.007$; Supplementary Figure 1a; Supplementary Table 2). We confirmed this positive correlation also at the protein level in the same cohort ( $r s=0.342$; $P<0.0001$; Supplementary Figure 1b), and by analyzing gene expression data from three publicly available TNBC data sets $\left(N=311 ; r_{\mathrm{S}}=0.360 ; P<0.0001\right.$; Supplementary Figure $1 c$; Supplementary Table 1).

Furthermore, considering the potential association between AXL expression and tumor immune response, we evaluated the clinical significance of global macrophage content in the internal cohort of TNBC patients. We found a higher content of CD68-positive macrophages in TNBC patients who experienced recurrence within 36 months after surgery compared with non-recurrent patients $(55.3 \%$ vs. $38.5 \%, P=0.045$; Figure 1a; Table 1). No statistically significant association between the presence of CD68positive macrophages and other clinicopathological parameters was, however, identified (Table 1). Furthermore, Kaplan-Meier analysis showed no prognostic relevance of CD68-positive macrophage count in terms of relapse-free survival (RFS) and overall survival (OS) in TNBC patients (Figure 1b).

AXL correlates with M2-polarized macrophages and is an independent prognostic marker in TNBC

To evaluate the potential role of $A X L$ in the regulation of cancerrelated inflammation in TNBC, we analyzed co-regulated genes
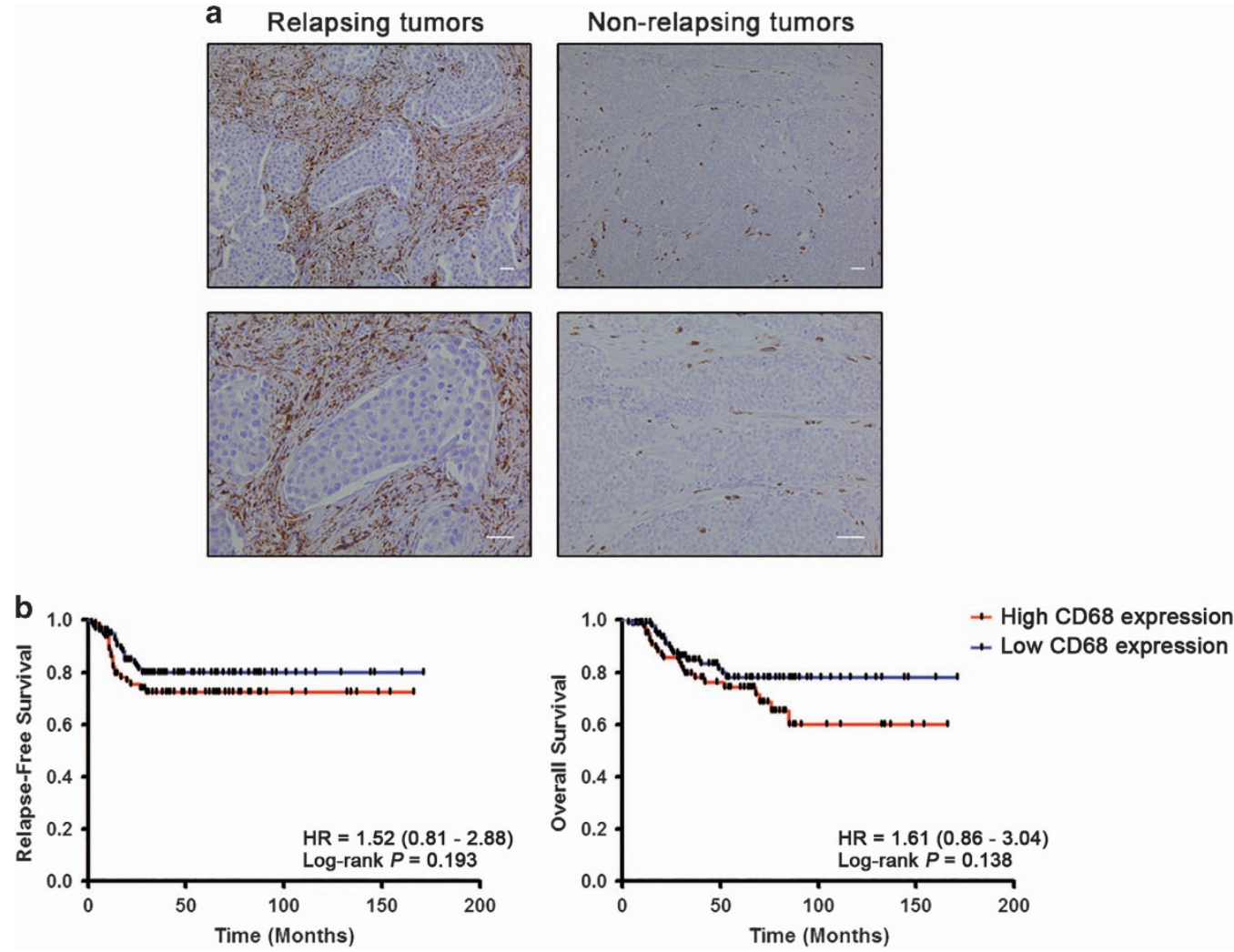

Figure 1. High infiltration of CD68-positive cells is associated with tumor relapse but not with survival in triple-negative breast cancer. (a) Representative immunohistochemical staining of CD68 in tumor samples from TNBC patients with recurrence (left panel) and without recurrence (right panel). Scale bars represent $50 \mu \mathrm{m}$. (b) Kaplan-Meier analysis for relapse-free survival (left panel) and overall survival (right panel) according to the content of CD68-positive cells in tumor stroma. TNBC patients $(N=203)$ were stratified by absent/moderate $(0-2)$ or dense (3) macrophage infiltration. Curves were compared using log-rank test. $P$ values and $\mathrm{HR}(95 \% \mathrm{Cl}$ in parentheses) are shown. $\mathrm{Cl}$, confidence interval; $\mathrm{HR}$, hazard ratio; TNBC, triple-negative breast cancer. 


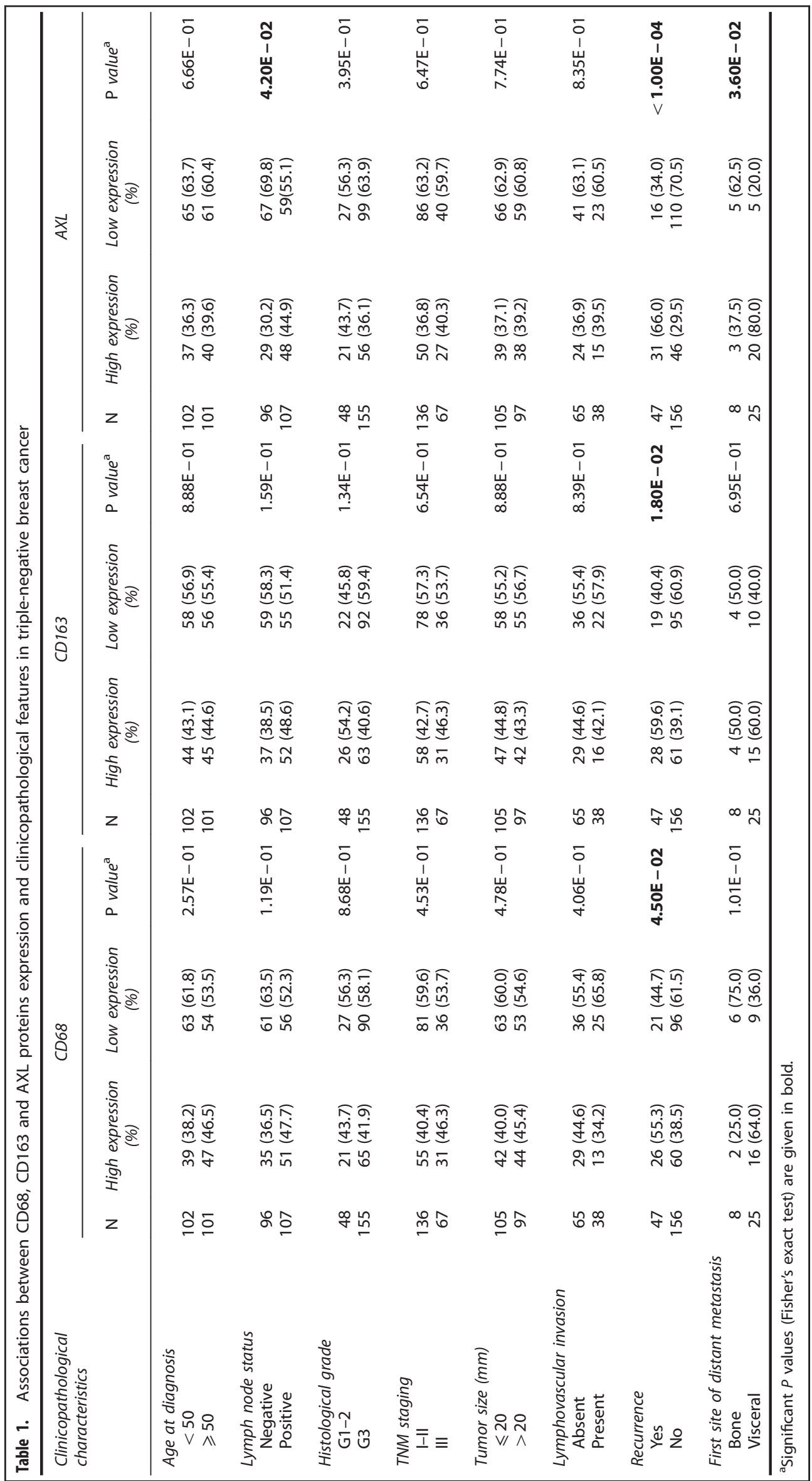


and pathways using the SEEK platform and the Ingenuity Pathway Analysis software. This analysis demonstrated that, besides its well-known role in EMT, $A X L$ is strongly co-expressed with genes involved in several immune functions (Supplementary Figure 2a; Supplementary Table 3), confirming AXL as a key factor of a gene network that influence both EMT and tumor-associated inflammation in TNBC.

Given the plasticity and heterogeneity of macrophages, we next investigated the role of $A X L$ in shaping the inflammatory tumor microenvironment in TNBC. Two distinct immune gene modules reflecting the polarization of anti-tumor M1 or pro-tumor M2 macrophages were assembled based on a literature review and analyzed using SEEK (Supplementary Figure 2b,c and Supplementary Table 4). We found that $A X L$ was consistently co-expressed with genes enclosed in the M2-related module (co-expression score $=1.013$; $P=0.013$ ), whereas it showed no relationship with M1-polarized macrophages in TNBC (Supplementary Figure 2b,c and Supplementary Table 5), suggesting that AXL expressed by tumor cells may be involved in the switch towards an M2 phenotype, thus sustaining the pro-tumor activity of TAMs.

To confirm these observations, we analyzed the expression of $A X L$ and the M2 macrophage marker CD163 in the internal cohort of 203 TNBC patients, which revealed a positive correlation between $A X L$ and the infiltration of CD163-positive cells $\left(r_{\mathrm{S}}=0.503 ; P<0.0001\right.$; Figure 2a). Furthermore, TNBC patients who experienced distant relapse had a significant higher expression of both $A X L$ and $C D 163$ compared with patients without recurrence $(P<0.0001 ; P=0.018$, respectively; Figure $2 \mathrm{~b}$ and $c$; Table 1). High levels of AXL expression were also associated with lymph node positivity $(P=0.042)$, and interestingly with metastasis to visceral organs as the first sites of distant recurrence $(P=0.036$; Table 1$)$.

To assess the prognostic value of AXL and CD163 in TNBC patients, we performed Kaplan-Meier and Cox univariate regression analyses in the internal cohort of TNBC patients $(N=203$; Figure $2 \mathrm{~d}$,e and Supplementary Table 6). We found that patients with high levels of AXL protein expression had significant shorter RFS and OS (log-rank $P=0.0002$; hazard ratio $(\mathrm{HR})=3.44 ; 95 \%$ confidence interval $(\mathrm{Cl}), 1.78-6.65$ for RFS; log-rank $P=0.0003$; $\mathrm{HR}=3.38 ; 95 \% \mathrm{Cl}, 1.75-6.50$ for OS; Figure $2 \mathrm{c}$ and Supplementary Table 6), whereas CD163 was associated with RFS only (log-rank $P=0.029 ; \mathrm{HR}=2.03 ; 95 \% \mathrm{Cl}, 1.08-3.83$; Figure $2 \mathrm{~d}$ and Supplementary Table 6). Cox multivariate regression analysis, adjusted for age at diagnosis, histological grade, lymph node status, tumor size, and tumor stage, demonstrated that only AXL expression remained an independent poor prognostic factor for RFS and OS in TNBC patients treated with adjuvant chemotherapy, along with lymph node status, whereas CD163 only retained a positive trend for reduced RFS (Table 2). Multivariate Cox analysis performed in an additional independent cohort of 95 chemotherapy-treated TNBC patients, and also analyzing $A X L$ as a continuous variable, confirmed the prognostic significance of AXL for both RFS and OS (Supplementary Table 7 and Supplementary Table 8).

We next assessed the prognostic value of $A X L$ by analyzing the gene expression data from 137 TNBC patients treated with adjuvant chemotherapy (Supplementary Table 1). We confirmed that $A X L$ expression was consistently associated with reduced RFS (log-rank $P=0.008 ; \mathrm{HR}=2.3 ; 95 \% \mathrm{Cl}, 1.20-4.30$; Supplementary Figure 3a). We also found that $A X L$ expression did not correlate with expression levels of the proliferation marker MKI67 (Pearson's coefficient, $r=0.014$; Supplementary Figure 3b).

AXL-overexpressing breast cancer cells and M2-like macrophages reciprocally interact in vitro

To characterize the expression of $A X L$ in vitro, we analyzed the expression of the tyrosine kinase, EMT (CDH1 and VIM), and basal (EGFR, KRT5, and KRT6A) markers in 15 breast cancer cell lines by quantitative real-time PCR (qRT-PCR; Figure 3a). We demonstrated that $A X L$ expression was higher in all triple-negative mesenchymal-like breast cancer cell lines, compared with luminal/epithelial cells (Figure 3a). High levels of $A X L$ expression were also found in two triple-negative basal-like cells (Figure 3a).

Then, we investigated the biological mechanisms underlying the cross-talk between $A X L$-expressing breast cancer cells and TAMs, and its impact on tumor progression and anticancer drug resistance. To identify the soluble factors potentially mediating the interaction between macrophages and breast cancer cells, we measured the release of several major cytokines/chemokines by macrophages exposed to the conditioned medium (CM) derived from the $A X L$-expressing breast cancer cell lines HCC38, MDAMB-231, and MDA-MB-436, and from the AXL-negative MCF-7 cells. We found that the medium of macrophages treated with the $\mathrm{CM}$ derived from $A X L$-expressing cells, especially from mesenchymallike cells, was enriched for tumor-promoting mediators, including CCL18, and IL-10, compared with that from macrophages treated with MCF-7-CM (Figure 3b). Conversely, the presence of AXLexpressing cells did not affect the release of $\mathrm{IL}-12$, which is commonly associated with the M1 phenotype (Figure $3 \mathrm{~b}$ ). Mesenchymal-like cells also induced an increased production of the AXL ligand Gas6 $(P<0.05$; Figure $3 b)$, and a positive trend was also observed for basal-like cells, although not reaching statistical significance (Figure 3b). Consistently, we found that Gas6 was significantly released from in vitro polarized M2-like macrophages, but not from M1-like cells ( $P=0.014$; Figure $3 c)$. These results may suggest that the interaction between M2 TAMs and mesenchymal TNBC cells could be partially modulated by the AXL/Gas6 signaling. To further investigate the ability of $A X L$-expressing TNBC cells to promote macrophage polarization towards a protumor phenotype, we analyzed the expression of the M2 specific markers CD206 and CD163 by flow cytometry (Figure 3d). AXLexpressing mesenchymal-like TNBC cells, but not MCF-7, were able to polarize macrophages towards an M2 phenotype (Figure $3 \mathrm{~d}$, upper panel). We also found that $A X L$-expressing basal-like cells can induce these phenotypic changes in macrophages, although to a lesser extent than mesenchymal-like cells, whereas the polarizing effect of $A X L$-negative basal-like cells was marginal (Supplementary Figure 4), suggesting that AXL may sustain the cancer inflammation cross-talk beyond its primary role in EMT. Noteworthy, the capability of MDA-MB-231 cells to change the expression of these surface markers was impaired by the selective inhibition of AXL with R428 (Figure 3d, lower panel). Consistently, we found that R428-treated MDA-MB-231 cells showed reduced expression of vimentin $(P=0.008), C C L 2(P=0.008)$, IL6 $(P=0.024)$, oncostatin $M(O S M, P=0.019)$, and TGFB2 $(P=0.029)$ as compared with untreated cells (Figure $3 e$ ), indicating that $A X L$ may contribute to the recruitment, and the polarization of macrophages into $\mathrm{M} 2$ cells by increasing the release of specific cytokines and chemokines.

Afterwards, we evaluated the relevance of the reciprocal crosstalk between M2 TAMs and cancer cells for tumor progression and chemotherapy response. We demonstrated that the $\mathrm{CM}$ from M2-polarized macrophages enhanced MDA-MB-231 cell migration $(P=0.020$; Figure $4 \mathrm{a}, \mathrm{b})$, and increased the resistance of MDAMB-231 and HCC38 TNBC cells to paclitaxel $(P=0.028 ; P=0.039$, respectively) and doxorubicin $(P=0.043 ; P=0.029$, respectively; Figure 4c). Importantly, AXL inhibition with R428 reduced the migratory capacity $(P=0.036$, Figure $4 a, b)$ and restored the sensitivity of MDA-MB-231 and $\mathrm{HCC} 38$ cells to paclitaxel $(P=0.012 ; \quad P=0.019$, respectively) and doxorubicin ( $P=0.027$; $P=0.020$, respectively; Figure $4 c$ ). In addition to $A X L$ inhibition, the treatment with R428 affected the activation of other oncogenic pathways, including AKT, ERK1/2, and SRC signaling, suggesting possible cross-talks and escape mechanisms to AXL inhibition in TNBC (Figure 4d). Collectively, these data suggest that the interaction between M2 TAMs and TNBC cells through AXL has 
a

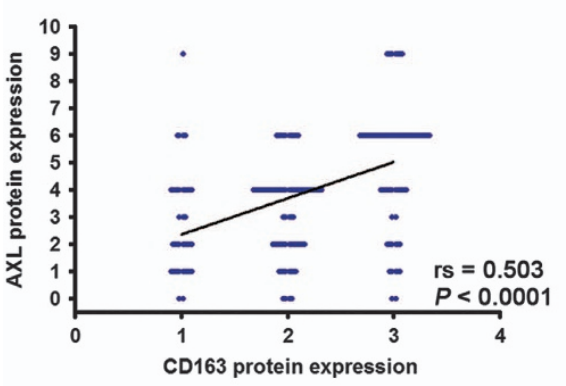

b

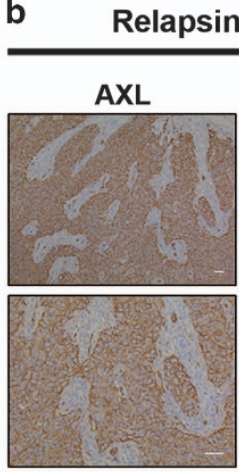

Non-relapsing tumors

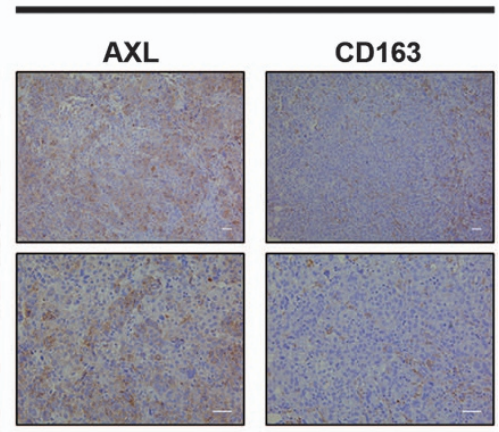

C
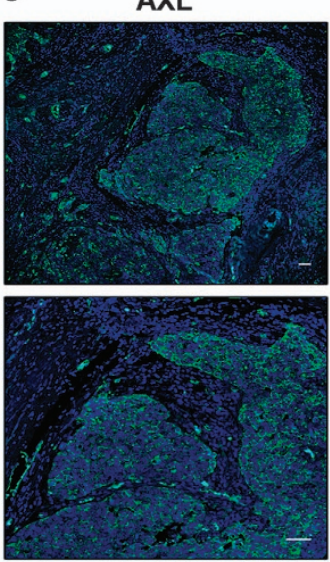

CD163
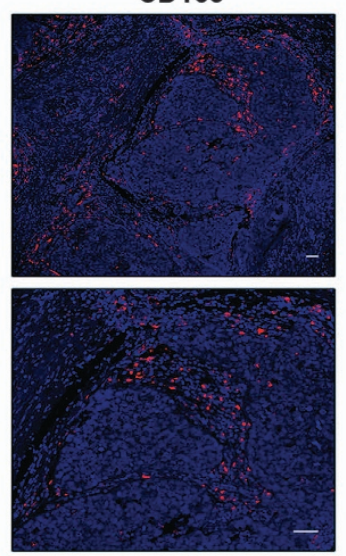

CD163

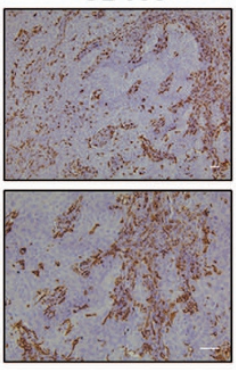

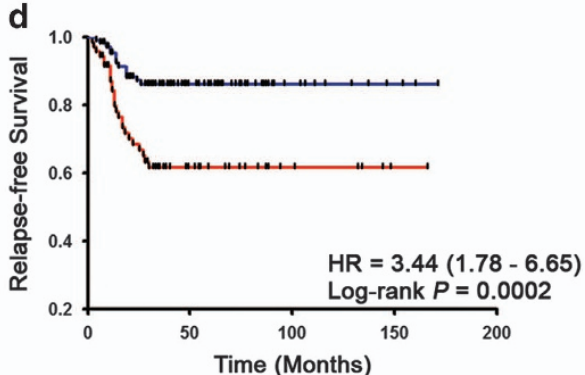

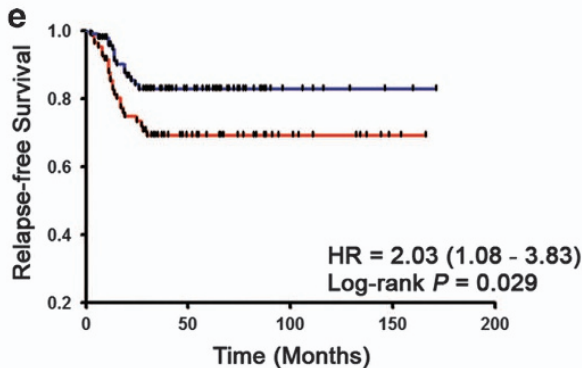

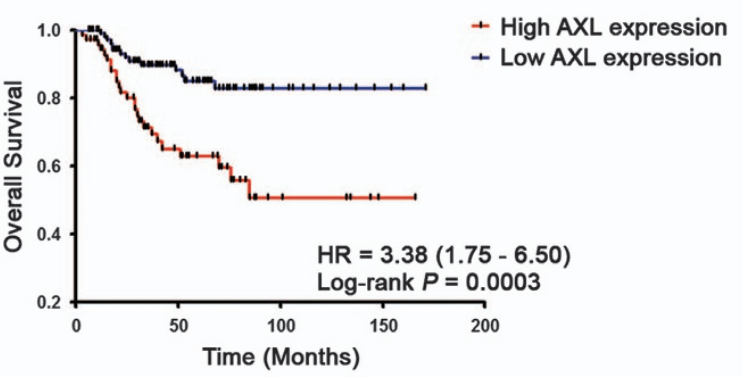

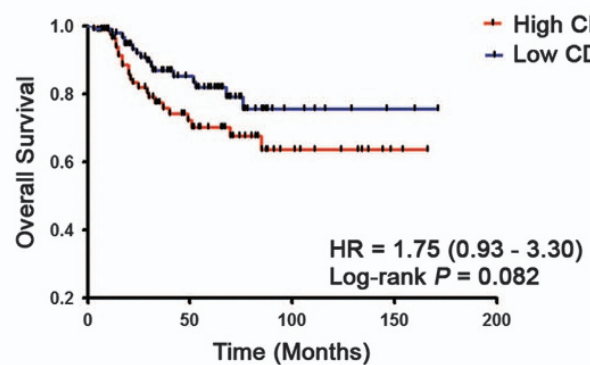

Figure 2. AXL expression correlates with the infiltration of CD163-positive cells in tumor stroma and is associated with survival in triplenegative breast cancer. (a) A scatter diagram shows a positive correlation between immunohistochemical staining of AXL and CD163 in TNBC $\left(N=203\right.$; Spearman's coefficient, $\left.r_{S}=0.503 ; P<0.0001\right)$. (b) Representative immunohistochemical staining of $A X L$ and $C D 163$ in tumor samples of serial sections from TNBC patients with recurrence (left panel) and without recurrence (right panel). Scale bars represent $50 \mu \mathrm{m}$. (c) Representative pictures of double immunofluorescent staining and confocal microscopy of TNBC sections, showing that AXL-expressing cancer cells (green) are in close contact with adjacent stromal TAMs (red). Scale bars represent $50 \mu \mathrm{m}$. (d) Kaplan-Meier analysis for relapsefree survival (left panel) and overall survival (right panel) according to AXL immunohistochemical score. TNBC patients $(N=203)$ were stratified by low (0-4) or high (6-9) AXL expression. (e) Kaplan-Meier analysis for relapse-free survival (left panel) and overall survival (right panel) according to the content of CD163-positive cells in tumor stroma. TNBC patients $(N=203)$ were stratified by absent/moderate $(0-2)$ or dense (3) CD163-positive macrophage infiltration. Curves were compared using log-rank test. $P$ values and $\mathrm{HR}(95 \% \mathrm{Cl}$ in parentheses) are shown. $\mathrm{Cl}$, confidence interval; $\mathrm{HR}$, hazard ratio; TNBC, triple-negative breast cancer. 
Table 2. Multivariate Cox regression analysis of AXL and CD163 for relapse-free survival and overall survival in triple-negative breast cancer $(N=203)$

\begin{tabular}{|c|c|c|c|c|c|c|}
\hline \multirow[t]{2}{*}{ Variable } & $H R$ & $95 \% \mathrm{Cl}$ & $\mathrm{P}$ value $\mathrm{a}^{\mathrm{a}}$ & $H R$ & $95 \% \mathrm{Cl}$ & $\mathrm{P}$ value $\mathrm{a}^{\mathrm{a}}$ \\
\hline & \multicolumn{3}{|c|}{ Relapse-free survival } & \multicolumn{3}{|c|}{ Overall survival } \\
\hline Age & 0.88 & $0.46-1.69$ & $7.11 \mathrm{E}-01$ & 1.08 & $0.57-2.07$ & $8.09 E-01$ \\
\hline Grade & 1.68 & $0.69-4.11$ & $2.53 E-01$ & 2.06 & $0.84-5.09$ & $1.16 \mathrm{E}-01$ \\
\hline Nodal status & 2.53 & $1.01-6.31$ & $4.72 \mathrm{E}-02$ & 2.62 & $1.05-6.55$ & $3.98 \mathrm{E}-02$ \\
\hline Tumor size & 1.33 & $0.69-2.58$ & $3.97 \mathrm{E}-01$ & 1.17 & $0.61-2.25$ & $6.30 E-01$ \\
\hline Age & 0.95 & $0.50-1.80$ & $8.72 \mathrm{E}-01$ & 1.16 & $0.61-2.20$ & $6.45 \mathrm{E}-01$ \\
\hline Grade & 1.68 & $0.68-4.13$ & $2.60 \mathrm{E}-01$ & 1.90 & $0.77-4.73$ & $1.65 \mathrm{E}-01$ \\
\hline Nodal status & 2.90 & $1.18-7.13$ & $2.02 E-02$ & 3.08 & $1.25-7.59$ & $1.43 E-02$ \\
\hline Tumor size & 1.36 & $0.70-2.65$ & $3.60 \mathrm{E}-01$ & 1.19 & $0.62-2.30$ & $5.99 \mathrm{E}-01$ \\
\hline Tumor stage & 1.54 & $0.74-3.22$ & $2.48 \mathrm{E}-01$ & 1.41 & $0.67-2.94$ & $3.66 \mathrm{E}-01$ \\
\hline
\end{tabular}

a

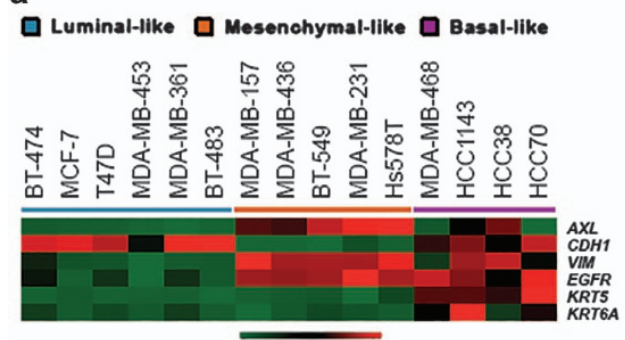

b

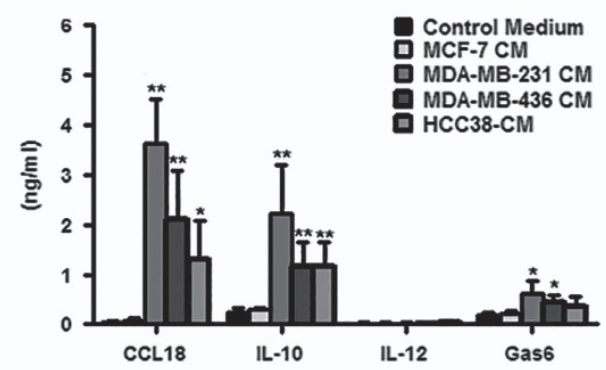

C

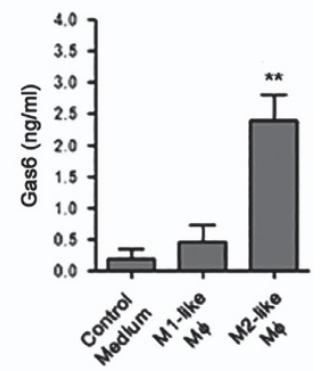

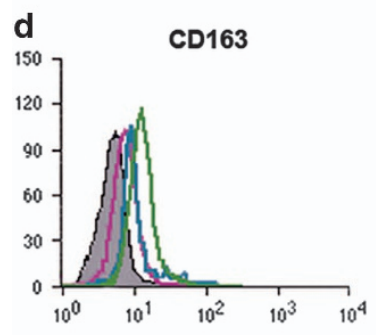

CD206

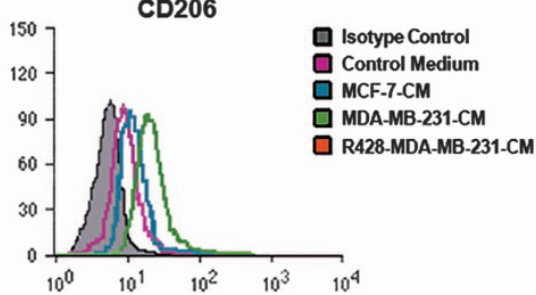

e
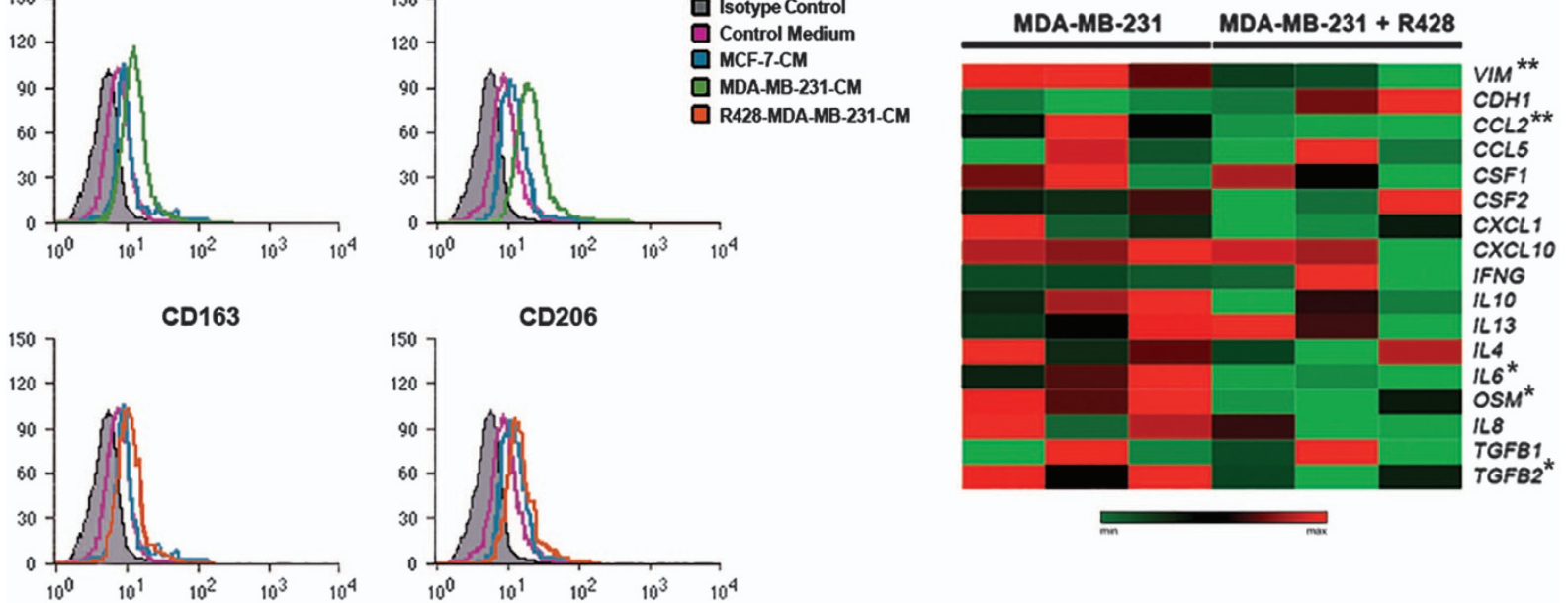

Figure 3. $A X L$-overexpressing breast cancer cells promote the polarization of $M 2$ macrophages. (a) Expression analysis of $A X L$, EMT (CDH1 and $V I M)$, and basal (EGFR, KRT5, and KRT6A) markers in 15 breast cancer cell lines by qRT-PCR. Gene expression levels were visualized in a heatmap. (b) Cytokine levels in the media of macrophages cultured in the absence or presence of MCF-7- or TNBC cell-derived conditioned medium (CM) measured by ELISA. $P$ values were obtained using a two-tailed Student's $t$-test (mean \pm s.d., $N=4$ experiments; ${ }^{*} P<0.05 ;{ }^{* *} P \leqslant 0.01$ ). (c) ELISA analysis of Gas6 in the medium of in vitro polarized human macrophages $(\mathrm{M} \varphi)$. $P$ values were obtained using a two-tailed Student's $t$-test (mean \pm s.d., $N=4$ experiments; ${ }^{*} P \leqslant 0.01$ ). (d) Flow cytometric analysis of the M2 markers CD163 and CD206 in human monocytes cultured in the absence (pink) or presence of MCF-7-CM (blue), MDA-MB-231-CM (green; upper panel), or R428-treated MDA-MB-231-CM (orange; lower panel) for 6 days. Gray histograms represent staining with isotype controls. The histograms are representatives of five independent experiments. (e) Heatmap showing the effect of R428 on the expression of EMT markers and relevant cytokines/chemokines in MDA-MB-231 cells (three biological replicates were shown). Significant genes were indicated with an asterisks $\left({ }^{*} P<0.05 ;{ }^{* *} P \leqslant 0.01\right)$. ELISA, enzyme-linked immunosorbent assay; EMT, epithelial-to-mesenchymal transition; TNBC, triple-negative breast cancer. 
Control Medium
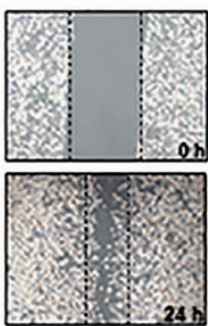

\section{.}

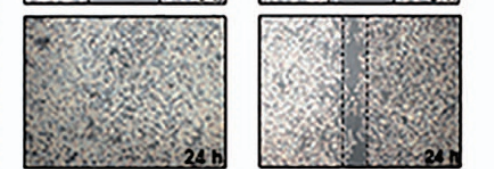

C
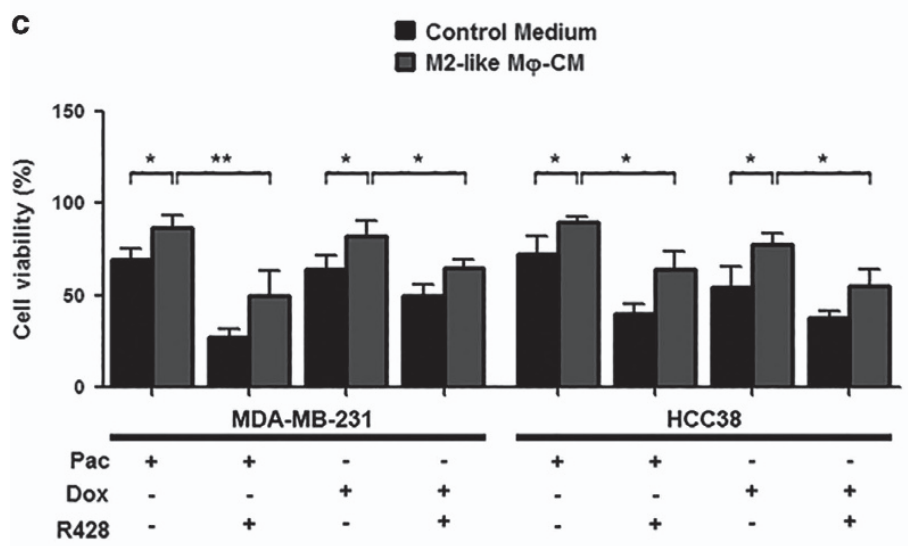

b

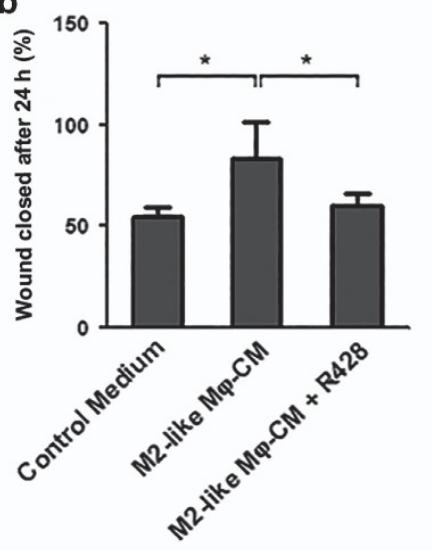

d

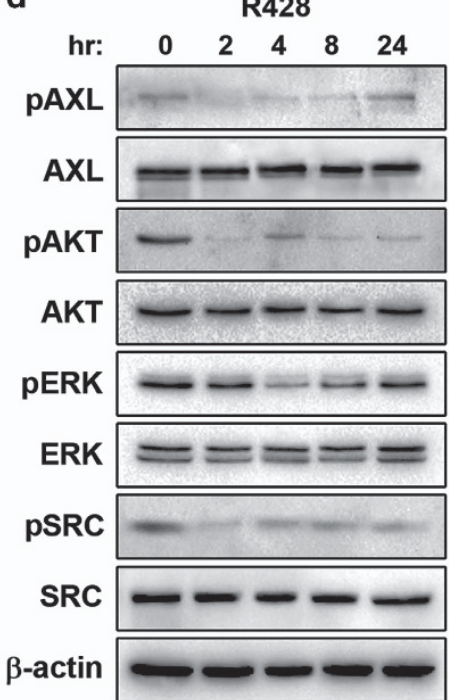

Figure 4. M2-polarized macrophages sustain tumor aggressiveness and influence drug sensitivity of $A X L$-overexpressing breast cancer cells. (a) Wound-healing assays were conducted with untreated or R428-treated MDA-MB-231 cells cultivated in the absence (control medium) or presence of conditioned medium (CM) derived from in vitro polarized M2 macrophages. (b) Statistical analysis of wound closure. Gap size at $0 \mathrm{~h}$ was set to $100 \%$ and percentage of closed wound was calculated after $24 \mathrm{~h}$. (c) M2-polarized macrophages increase the resistance of HCC38 and MDA-MB-231 cells to paclitaxel (Pac) and doxorubicin (Dox) treatments compared with cells treated with control medium, whereas the selective inhibition of AXL with R428 restores drug sensitivity in TNBC cells. (d) Western blot with the indicated antibodies of lysates from MDA-MB-231 cells treated with $1 \mu \mathrm{mol} / \mathrm{I}$ R428 at different time points. $\beta$-Actin was used as a loading control. All $P$ values were obtained using a two-tailed Student's $t$-test (mean \pm s.d., $N=3$ experiments; ${ }^{*} P<0.05 ;{ }^{*} P \leqslant 0.01$ ).

an important role in supporting tumor progression and chemoresistance.

\section{DISCUSSION}

The tumor microenvironment is of paramount importance in breast cancer progression, and accumulating evidence indicates an emerging role of the cross-talk signaling between mesenchymal cancer cells and TAMs. In fact, EMT and TAMs provide invasive and metastatic capabilities to tumor cells and modulate the tumor microenvironment, leading to the suppression of anticancer immune response, and limiting the effects of cytotoxic chemotherapy. ${ }^{6-11}$ TNBC, which is often characterized by the presence of both EMT and TAMs, is a good model to explore potential molecular markers maintaining the biological intersections between these two signalings. ${ }^{9-12}$ In this study, we demonstrated that the receptor AXL was the most significant EMTrelated kinase associated with macrophage cells in the tumor stroma of TNBC. This receptor is emerging as an important effector of the EMT program, and its activation is responsible for triggering important oncogenic pathways, such as PI3K/AKT/mTOR, NF-KB, EGFR, and MAPK cascades, involved in cell proliferation, survival, and invasion. ${ }^{21-26}$ In line with different studies on human cancer, we found that the increased expression of AXL significantly correlated with poor outcome in TNBC. ${ }^{21-28}$ High levels of AXL were associated with reduced RFS and OS in TNBC patients treated with anthracycline-taxane-based adjuvant chemotherapy. Interestingly, AXL overexpression was associated with distant tumor recurrence, particularly to visceral organs, indicating a specific route of tumor dissemination for a subset of these tumors. $A X L$ has been previously shown to be one of the most differentially expressed genes in the mesenchymal stem-like subtype compared with other subgroups of TNBC. ${ }^{29}$ Our data demonstrated that the expression of $A X L$ was a specific feature of TNBC cells, especially those with mesenchymal features, sustaining the key role of AXL in the activation of the EMT program, and in mediating cancer cell aggressiveness. ${ }^{21-24,30}$ Furthermore, we showed that AXL was involved in the modulation of several immune pathways, including leukocyte migration and chemotaxis, macrophage activation, and agranulocyte adhesion and 
diapedesis, further supporting the biological relevance of the interaction between $A X L$-expressing cancer cells and cancerrelated immune responses in TNBC. ${ }^{9-15}$

Previous studies reported that CD68-positive TAMs are a potential prognostic marker in breast cancer. ${ }^{17,22,31,32}$ However, we found that CD68-positive macrophages in tumor stroma mildly correlated with tumor relapse and were not significantly associated with TNBC patients outcome. Therefore, given potential differences in the evaluation of protein expression among studies, the clinical significance and utility of CD68 expression in TNBC remains uncertain. These data also highlight the concept that CD68 probably does not accurately reflect the presence and function of distinct macrophage subpopulations within the tumor stroma, at least in breast cancer. ${ }^{18,33,34}$ Consequently, our results advocate the importance to further evaluate the biological role and functions of different macrophage subpopulations in breast cancer. Indeed, macrophages exhibit remarkable functional and phenotypic plasticity, with activated M2-like cells displaying tumor-promoting activities. ${ }^{14,22}$ Accordingly, most human tumors exhibit TAMs with an M2-like phenotype, promoting EMT, and contributing to tumor progression and drug resistance. ${ }^{11-20,35}$ In agreement with these reports, we found that the massive presence of $\mathrm{M} 2$ macrophages correlated with an aggressive behavior of TNBC. Moreover, AXL was significantly co-expressed with genes associated with M2 macrophages, and positively correlated with the infiltration of CD163-positive M2 cells, suggesting an important relationship between $A X L$-expressing cells and TAMs with pro-tumor activity in a subgroup of TNBC.

The cross-talk between mesenchymal cancer cells and tumor microenvironment is recognized as a key factor in tumor progression in several tumors. ${ }^{10-12,14,30}$ However, we demonstrated that CD163-positive M2 TAMs did not retain their prognostic significance in multivariate analysis. Our data suggest that the CD163-positive embedded stromal cells likely have a role in sustaining the aggressiveness of cancer cells, but the presence of pro-tumor macrophages alone may not be sufficient to affect the outcome of TNBC patients. Although the mechanisms underlying the link between cancer cells and TAMs are complex and difficult to dissect in vitro, we observed the reciprocal nature of this interaction sustained by $\mathrm{AXL}$. Our data show that $A X L$-positive TNBC cells with mesenchymal traits activate human macrophages to an M2-like phenotype, modulating a specific pattern of pro-tumor cytokines and chemokines. Indeed, mesenchymal-like cells were able to increase the release of CCL18 and IL-10, which are known macrophage-derived mediators of metastatic dissemination and resistance to chemotherapy in breast cancer. ${ }^{36,37}$ Furthermore, we demonstrated that the selective inhibition of AXL with R428 impaired the ability of mesenchymal cells to induce the polarization of macrophages, by reducing the release of $C C L 2, I L-6$, oncostatin $M$, and TGF- $\beta$, which are well-known inducers of the M2 phenotype. ${ }^{13,38}$ In addition, AXL may be also involved in the recruitment of macrophages at the tumor site, increasing the production of CCL2 that functions as a monocyte chemoattractant protein. ${ }^{39}$ Noteworthy, we found that $A X L$-expressing mesenchymal-like cells were also capable of inducing the release of the AXL ligand Gas6, which is selectively secreted by M2-type macrophages. Our results suggest that the AXL/Gas6 signaling may have a role in modulating the interaction between mesenchymal TNBC cells and M2 TAMs, and support previous findings, indicating that tumor cells induced infiltrating TAMs to increase the production of Gas6, promoting cell growth and metastasis in different cancer models. ${ }^{40}$ Even though AXL can be activated by Gas6, alternative ligand-independent mechanisms, including the interaction with EGFR, which is frequently express in TNBC, have been demonstrated. ${ }^{1,24-26}$ Therefore, although the biological modalities of AXL action should be further investigated, our results suggest that TNBC cells with mesenchymal features may 'educate' infiltrating TAMs to support tumor progression, and that targeting AXL may be a novel strategy to reduce both EMT and the pro-tumor activity of TAMs in TNBC.

Finally, we demonstrated that the presence of M2-polarized macrophages enhanced the migratory potential and chemoresistance of TNBC cells, whereas the selective pharmacological inhibition of AXL was able to drastically reduce cell aggressiveness, and to restore response to chemotherapeutic drugs, including taxanes and anthracyclines. These results are in agreement with recent findings showing that infiltrating macrophages reduced the primary breast tumor drug response, and that R428 enhanced the efficacy of anti-mitotic drugs in mesenchymallike lung and breast cancer cells. ${ }^{37,41}$ Noteworthy, AXL inhibition also affected the activation of other oncogenic pathways, providing the evidence of cross-talk signaling between different pathways and the activation of compensatory feedback networks. We showed that $A X L$ was also expressed in basal-like breast cancer cells. Even though the effect of $A X L$-expressing basal-like cells on macrophage polarization was mild compared with that of mesenchymal-like cells, TAMs were equally able to induce chemoresistance in both TNBC models. These findings suggest that, beyond the role of mesenchymal-like cells in supporting the crosstalk with TAMs, AXL inhibition could be a potential therapeutic strategy for a broader range of patients with $A X L$-expressing TNBC.

Our study has some limitations. Even though we provide evidence of the involvement of $A X L$ kinase in macrophage polarization, the specific requirement of $A X L$ for the interaction with TAMs, the identification of the soluble mediators of this crosstalk, and the potentially distinct biological role of different subtypes of TNBC cells warrant further studies. Given the retrospective nature of this study, and to determine the potential heterogeneity of treatment effects associated with AXL functions, our findings would need to be further validated in a prospective clinical trial. Despite these limitations, our results suggest that AXL is a prognostic indicator of outcome in TNBC treated with chemotherapy. Furthermore, AXL supports the pro-tumor activity of M2-type macrophages, inducing tumor progression and resistance to chemotherapy. Overall, our data provide support for the use of AXL targeted therapy to reduce tumor aggressiveness, overcome chemotherapy resistance, and impair the crosstalk between cancer cells and TAMs, in patients with TNBC overexpressing AXL.

\section{MATERIALS AND METHODS}

\section{Patients' cohorts and tumor samples}

Formalin-fixed, paraffin-embedded (FFPE) tissues were retrospectively collected from 203 patients with histologically confirmed invasive ductal TNBC, who underwent surgery at Humanitas Clinical and Research Institute (Rozzano-Milan, Italy) from 2006 to 2011. To validate our findings, an additional independent cohort of TNBC samples $(N=95)$ who underwent surgery at Humanitas Oncology Center of Catania (Catania, Italy; $N=59$ ) and Semmelweis University Hospital (Budapest, Hungary; $N=36$ ) from 1999 to 2008 was used. Estrogen, progesterone, and HER2 status were centrally assessed by immunohistochemistry and/or fluorescence in situ hybridization in $\sim 90 \%$ of patients at Humanitas Clinical and Research Institute. The study was approved by the ethical committees of the Italian and Hungarian Institutions. The REporting of tumor MARKer Studies (REMARK) guidelines were followed in reporting results of this study. ${ }^{42}$ All patients were treated with adjuvant anthracycline-taxane-based chemotherapy. Clinical characteristics of patients included in this study are presented in Supplementary Table 1.

Immunohistochemistry, immunofluorescence, and evaluation of staining

FFPE sections $(3 \mu \mathrm{m})$ from TNBC patients included in the discovery $(N=203)$ and validation $(N=95)$ cohorts were incubated with AXL (R\&D Systems, Minneapolis, MN, USA), CD163 (Novocastra, Newcastle, UK), and CD68 (Dako, Glostrup, Denmark) antibodies for $1 \mathrm{~h}$ at room temperature. CD68 and CD163 staining in the tumor stroma was scored using a 
four-tiered system ranging from 0 (absent) to 3 (dense). ${ }^{43} \mathrm{AXL}$ staining was scored semiquantitatively, as previously described. ${ }^{21}$ In brief, intensity was recorded as 0 (no staining), 1 (weak staining), 2 (moderate staining), or 3 (strong staining), and the proportion of positive tumor cells was defined as the following: $0<1 \% ; 1=1-9 \% ; 2=10-49 \%$; and $3 \geqslant 50 \%$. A composite staining index was calculated by multiplying the intensity by the percentage of positive cells, and patients were stratified by low (0-4) or high (6-9) AXL expression for statistical analyses. The optimal cut-off point was determined by maximizing the sum of sensitivity and specificity. AXL expression was also evaluated as a continuous variable. For immunofluorescence, sections were incubated with AXL and CD163 primary antibodies, and then with donkey anti-goat Alexa 488-conjugated (Life Technologies, Carlsbad, CA, USA) and donkey anti-mouse Alexa-647conjugated (Life Technologies) antibodies. Slides were counterstained with 4,6-diamidino-2-phenylindole (DAPI). Images were captured using an Olympus BX53 or Olympus Fluoview FV1000 laser scanning confocal microscope (Olympus, Tokyo, Japan). Detailed procedures for immunohistochemistry and immunofluorescence are described in Supplementary Methods.

\section{Expression analysis by quantitative reverse-transcription PCR}

On the basis of a comprehensive literature review, we selected the 30 most functionally relevant kinases associated with EMT in breast cancer (Supplementary Table 2), and evaluated their expression by qRT-PCR, as described in Supplementary Methods. We also assessed the expression of conventional EMT (CDH1 and VIM), and basal (EGFR, KRT5, and KRT6A) markers, and of a panel of cytokines/chemokines in breast cancer cell lines. The expression of selected genes was evaluated using TaqMan probes from Applied Biosystems (Foster City, CA, USA), following the manufacturer's guidelines (Supplementary Table 9).

Gene expression normalization and molecular subtype definition Publicly available gene expression data from 311 TNBC patients were collected (Supplementary Table 1) and used for correlative analysis. An additional cohort of 137 TNBC patients treated with adjuvant chemotherapy was analyzed to validate our findings from the discovery phase (Supplementary Table 1). Detailed in silico analyses are reported in Supplementary Methods.

Cell cultures, treatments, and preparation of tumor-conditioned media

Breast cancer cell lines (BT-474, BT-483, BT-549, HCC38, HCC70, HCC1143, Hs578T, MCF-7, MDA-MB-157, MDA-MB-453, MDA-MB-231, MDA-MB-361, MDA-MB-436, MDA-MB-468, and T47D) were obtained from the American Type Culture Collection (Manassas, VA, USA), and grown according to the standard protocols at $37^{\circ} \mathrm{C}$ with $5 \% \mathrm{CO}_{2}$. Paclitaxel, doxorubicin, and R428 (Selleck Chemicals, Houston, TX, USA) were dissolved in dimethyl sulfoxide. Cells were treated with paclitaxel $(25 \mathrm{nmol} / \mathrm{l})$, doxorubicin $(1 \mu \mathrm{mol} / \mathrm{l})$, R428 $(1 \mu \mathrm{mol} / \mathrm{l})$, or control vehicle. Once grown to sub-confluence, cells were serum starved and incubated with fresh medium for $24 \mathrm{~h}$. CM were collected and filtered at $0.2 \mu \mathrm{m}$.

\section{Macrophages differentiation}

Human monocytes were obtained from normal donor buffy coat by two-step gradient centrifugation and then polarized. Detailed procedures are described in Supplementary Methods. Freshly isolated human monocytes were also cultured in the absence or presence of 30\% CM from HCC38, MCF-7, MDAMB-231, MDA-MB-436, or MDA-MB-468 for 6 days, as previously described. ${ }^{12,44}$

\section{Flow cytometry}

Macrophages were treated as indicated in the text, and analyzed by flow cytometry on a FACS Canto flow cytometer (BD Biosciences, San Jose, CA, USA). Human FcRs were blocked using $1 \%$ human serum in phosphatebuffered saline. Cells were washed, resuspended in FACS buffer $(0.5 \%$ bovine serum albumin and $0.05 \% \mathrm{NaN}_{3}$ in phosphate-buffered saline), and stained with fluorochrome-conjugated monoclonal antibodies against CD163 and CD206, or appropriate isotype controls (BD Biosciences).

\section{Enzyme-linked immunosorbent assay}

The levels of CCL18, IL-10, IL-12, and growth arrest-specific 6 (Gas6) in macrophage supernatants were measured by commercially available ELISA kits according to manufacturer's instructions (R\&D Systems). All experiments were performed with three wells for each condition and repeated four times.

Cell viability and wound-healing assays

Viable cells were identified using the 3-[4,5-dimethylthiazol-2-yl]-2, 5-diphenyltetrazolium bromide assay (Sigma Aldrich, Milan, Italy), as previously described. ${ }^{45}$ For the wound-healing assay, breast cancer cells were seeded in six-well plates, and then scraped with a pipette tip. A detailed description of the assays can be found in Supplementary Methods.

\section{Western blotting}

Immunodetection of proteins was performed using standard protocols. Further details on immunoblotting are provided in Supplementary Methods. The phospho-AXL (Tyr779) and AXL antibodies were purchased from R\&D Systems. The phospho-AKT (Ser473), AKT, phospho-ERK1/2 (Thr202/Tyr204), ERK1/2, phospho-SRC (Tyr416), SRC, and $\beta$-actin were from Cell Signaling Technology (Danvers, MA, USA).

\section{Statistical analysis}

Differences between two groups were determined using the Student's $t$-test. Spearman's rank and Pearson's linear correlation tests were used to evaluate the correlation between variables. Clinicopathological associations were tested using Fisher's exact test. Co-expression and enrichment analyses were performed using the Search-based Exploration of Expression Compendium (SEEK; http://seek.princeton.edu). ${ }^{46}$ Pathway analysis was performed using Ingenuity Pathway Analysis software (Qiagen, Redwood City, CA, USA). Survival analyses were performed by the Kaplan-Meier method, log-rank test (Mantel-Cox), and Cox univariate proportional hazard model. Multivariate Cox proportional hazard regression analysis was adjusted for relevant clinical covariates, including age at diagnosis, histological grade, lymph node status, tumor size, and tumor stage. $P$ values were corrected using the Bonferroni or the Benjamini-Hochberg methods as indicated in the text. All tests were two-sided and the level of statistical significance was set at $P<0.05$. Statistical analyses were performed using GraphPad Prism version 5 (GraphPad Software, La Jolla, CA, USA), Epi Info version 7 (CDC, Atlanta, GA, USA), and R software (http:// www.r-project.org). Further details on statistical methods are described in Supplementary Methods.

\section{Availability of data and materials}

Information on the publicly available breast cancer data sets used in this study is provided in Supplementary Table 1.

\section{ACKNOWLEDGMENTS}

We are grateful to all subjects working at the Breast Units of Humanitas Hospitals (Milan and Catania, Italy) for their cooperation in collecting samples. A. Sica for helpful suggestions about in vitro data. This study was supported by Associazione Italiana Ricerca sul Cancro (grant 6251 to LS); Fondazione Italiana Ricerca sul Cancro (FIRC fellowship 18328 to GB); Associazione Italiana Ricerca sul Cancro (AIRC and AIRC 5x1000; AM); La Caixa Foundation and Redes Temáticas de Investigación en Cáncer (RD12/0036/0072 to RR).

\section{CONTRIBUTIONS}

Conception and design: LS. Development of methodology: GB, CR, and LS. Acquisition of data: BA, GB, MC, LDT, NK, JK, AL, CR, JSR-F, MR, RR, LS, MAS, BS, TT, and RT. Analysis and interpretation of data: GB, LDT, BG, AM, JSR-F, MR, RR, and LS. Writing, review, and/or revision of the manuscript: all authors. Administrative, technical, or material support: GB, CR, LS, and A.S. Study supervision: LS.

\section{COMPETING INTERESTS}

The authors declare no conflict of interest.

\section{REFERENCES}

1. Carey, L., Winer, E., Viale, G., Cameron, D. \& Gianni, L. Triple-negative breast cancer: disease entity or title of convenience? Nat. Rev. Clin. Oncol. 7, 683-692 (2010). 
2. Foulkes, W. D., Smith, I. E. \& Reis-Filho, J. S. Triple-negative breast cancer. N. Engl. J. Med. 363, 1938-1948 (2010).

3. Metzger-Filho, O. et al. Dissecting the heterogeneity of triple-negative breast cancer. J. Clin. Oncol. 30, 1879-1887 (2012).

4. Turner, N. C. \& Reis-Filho, J. S. Tackling the diversity of triple-negative breast cancer. Clin. Cancer Res. 19, 6380-6388 (2013).

5. Perou, C. M. Molecular stratification of triple-negative breast cancers. Oncologist 16, 61-70 (2011).

6. Drasin, D. J., Robin, T. P. \& Ford, H. L. Breast cancer epithelial-to-mesenchymal transition: examining the functional consequences of plasticity. Breast Cancer Res. 13, 226 (2011).

7. De Craene, B. \& Berx, G. Regulatory networks defining EMT during cancer initiation and progression. Nat. Rev. Cancer 13, 97-110 (2013).

8. Lamouille, S., Xu, J. \& Derynck, R. Molecular mechanisms of epithelialmesenchymal transition. Nat. Rev. Mol. Cell Biol. 15, 178-196 (2014).

9. Jung, H. Y., Fattet, L. \& Yang, J. Molecular pathways: linking tumor microenvironment to epithelial-mesenchymal transition in metastasis. Clin. Cancer Res. 21, 962-968 (2015).

10. Reiman, J. M., Knutson, K. L. \& Radisky, D. C. Immune promotion of epithelialmesenchymal transition and generation of breast cancer stem cells. Cancer Res. 70, 3005-3008 (2010).

11. Su, S., Wu, W., He, C., Liu, Q. \& Song, E. Breaking the vicious cycle between breast cancer cells and tumor-associated macrophages. Oncoimmunology 3, e953418 (2014).

12. Su, S., Liu, Q., Chen, J., Chen, J., Chen, F., He, C. et al. A positive feedback loop between mesenchymal-like cancer cells and macrophages is essential to breast cancer metastasis. Cancer Cell 25, 605-620 (2014).

13. Williams, C. B., Yeh, E. S. \& Soloff, A. C. Tumor-associated macrophages: unwitting accomplices in breast cancer malignancy. npj Breast Cancer 2, 15025 (2016).

14. Lu, H. et al. A breast cancer stem cell niche supported by juxtacrine signalling from monocytes and macrophages. Nat. Cell Biol. 16, 1105-1117 (2014).

15. DeNardo, D. G. et al. Leukocyte complexity predicts breast cancer survival and functionally regulates response to chemotherapy. Cancer Discov. 1, 54-67 (2011).

16. Mantovani, A. \& Allavena, P. The interaction of anticancer therapies with tumor-associated macrophages. J. Exp. Med. 212, 435-445 (2015).

17. Jinushi, M. et al. Tumor-associated macrophages regulate tumorigenicity and anticancer drug responses of cancer stem/initiating cells. Proc. Natl Acad. Sci. USA 108, 12425-12430 (2011).

18. Ruffell, B. \& Coussens, L. M. Macrophages and therapeutic resistance in cancer. Cancer Cell 27, 462-472 (2015)

19. Pollard, J. W. Tumour-educated macrophages promote tumour progression and metastasis. Nat. Rev. Cancer 4, 71-78 (2004).

20. Galluzzi, L., Senovilla, L., Zitvogel, L. \& Kroemer, G. The secret ally: immunostimulation by anticancer drugs. Nat. Rev. Drug Discov. 11, 215-233 (2012).

21. Gjerdrum, C. et al. Axl is an essential epithelial-to-mesenchymal transitioninduced regulator of breast cancer metastasis and patient survival. Proc. Natl Acad. Sci. USA 107, 1124-1129 (2010).

22. Graham, D. K., DeRyckere, D., Davies, K. D. \& Earp, H. S. The TAM family: phosphatidylserine sensing receptor tyrosine kinases gone awry in cancer. Nat. Rev. Cancer 14, 769-785 (2014).

23. Paccez, J. D., Vogelsang, M., Parker, M. I. \& Zerbini, L. F. The receptor tyrosine kinase Axl in cancer: biological functions and therapeutic implications. Int. J. Cancer 134, 1024-1033 (2014).

24. Asiedu, M. K. et al. AXL induces epithelial-to-mesenchymal transition and regulates the function of breast cancer stem cells. Oncogene 33, 1316-1324 (2014).

25. Meyer A. S., Miller M. A., Gertler F. B., Lauffenburger D. A. The receptor AXL diversifies EGFR signaling and limits the response to EGFR-targeted inhibitors in triple-negative breast cancer cells. Sci. Signal 6, ra66 (2013).

26. Elkabets, M. et al. AXL mediates resistance to PI3Ka inhibition by activating the EGFR/PKC/mTOR axis in head and neck and esophageal squamous cell carcinomas. Cancer Cell 27, 533-546 (2015).

27. Brand, T. M. et al. AXL Is a Logical Molecular Target in Head and Neck Squamous Cell Carcinoma. Clin. Cancer Res. 21, 2601-2612 (2015).
28. Dunne, P. D. et al. AXL is a key regulator of inherent and chemotherapy-induced invasion and predicts a poor clinical outcome in early-stage colon cancer. Clin. Cancer Res. 20, 164-175 (2014).

29. Lehmann, B. D. et al. Identification of human triple-negative breast cancer subtypes and preclinical models for selection of targeted therapies. J. Clin. Invest. 121, 2750-2767 (2011).

30. Del Pozo Martin, Y. et al. Mesenchymal cancer cell-stroma crosstalk promotes niche activation, epithelial reversion, and metastatic colonization. Cell Rep. 13, 2456-2469 (2015)

31. Mahmoud, S. M. et al. Tumor-infiltrating macrophages and clinical outcome in breast cancer. J. Clin. Pathol. 65, 159-163 (2012).

32. Tiainen, S. et al. High numbers of macrophages, especially M2-like (CD163-positive), correlate with hyaluronan accumulation and poor outcome in breast cancer. Histopathology 66, 873-883 (2015).

33. Ruffell, B. et al. Leukocyte composition of human breast cancer. Proc. Natl Acad. Sci. USA 109, 2796-2801 (2012).

34. Mukhtar, R. A., Nseyo, O., Campbell, M. J. \& Esserman, L. J. Tumor-associated macrophages in breast cancer as potential biomarkers for new treatments and diagnostics. Expert Rev. Mol. Diagn. 11, 91-100 (2011).

35. Qian, B. Z. \& Pollard, J. W. Macrophage diversity enhances tumor progression and metastasis. Cell 141, 39-51 (2010).

36. Chen, J. et al. CCL18 from tumor-associated macrophages promotes breast cancer metastasis via PITPNM3. Cancer Cell 19, 541-555 (2011).

37. Ruffell, B. et al. Macrophage IL-10 blocks CD8+ T cell-dependent responses to chemotherapy by suppressing IL-12 expression in intratumoral dendritic cells. Cancer Cell 26, 623-637 (2014)

38. Tripathi, C. et al. Macrophages are recruited to hypoxic tumor areas and acquire a pro-angiogenic M2-polarized phenotype via hypoxic cancer cell derived cytokines Oncostatin M and Eotaxin. Oncotarget 5, 5350-5368 (2014).

39. Kitamura, T. et al. CCL2-induced chemokine cascade promotes breast cancer metastasis by enhancing retention of metastasis-associated macrophages. J. Exp. Med. 212, 1043-1059 (2015).

40. Loges, S. et al. Malignant cells fuel tumor growth by educating infiltrating leukocytes to produce the mitogen Gas6. Blood 115, 2264-2273 (2010).

41. Wilson, C. et al. AXL inhibition sensitizes mesenchymal cancer cells to antimitotic drugs. Cancer Res. 74, 5878-5890 (2014).

42. McShane, L. M. et al. Reporting recommendations for tumor marker prognostic studies (REMARK). J. Natl Cancer Inst. 97, 1180-1184 (2005).

43. Medrek, C., Pontén, F., Jirström, K. \& Leandersson, K. The presence of tumor associated macrophages in tumor stroma as a prognostic marker for breast cancer patients. BMC Cancer 12, 306 (2012).

44. Solinas, G. et al. Tumor-conditioned macrophages secrete migration-stimulating factor: a new marker for M2-polarization, influencing tumor cell motility. J. Immunol. 185, 642-652 (2010).

45. Györffy, B. et al. TP53 mutation-correlated genes predict the risk of tumor relapse and identify MPS1 as a potential therapeutic kinase in TP53-mutated breast cancers. Mol. Oncol. 8, 508-519 (2014).

46. Aure, M. R. et al. Individual and combined effects of DNA methylation and copy number alterations on miRNA expression in breast tumors. Genome Biol. 14, R126 (2013).

This work is licensed under a Creative Commons Attribution 4.0 International License. The images or other third party material in this article are included in the article's Creative Commons license, unless indicated otherwise in the credit line; if the material is not included under the Creative Commons license, users will need to obtain permission from the license holder to reproduce the material. To view a copy of this license, visit http://creativecommons.org/licenses/ by/4.0/

(c) The Author(s) 2016

Supplementary Information accompanies the paper on the npj Breast Cancer website (http://www.nature.com/npjbcancer) 